\title{
Three-dimensional holographic disks
}

\author{
Hsin-Yu Sidney Li and Demetri Psaltis
}

\begin{abstract}
We describe optical disks that store data holographically in three dimensions by using either angle multiplexing or wavelength multiplexing. Data are stored and retrieved in parallel blocks or pages, and each page consists of approximately $10^{6}$ bits. The storage capacity of such disks is derived as a function of disk thickness, pixel size, page size, and scanning parameters. The optimum storage density is approximately $120 \mathrm{bits} / \mu \mathrm{m}^{2}$.
\end{abstract}

\section{Introduction}

Holographic storage of data in three-dimensional media such as photorefractive crystals can provide high storage density and fast parallel access to the stored information. Such memories were investigated extensively in the early 1960's. ${ }^{1-5}$ Even though these early efforts produced remarkable results, they never found practical application largely because of material limitations such as low sensitivity, fanning, and hologram decay. Interest in holographic threedimensional (3-D) memories has been revitalized in recent years for a variety of reasons, including significant advances in recording materials (photorefractive and photopolymers), dramatic improvements in all optical devices (lasers, spatial light modulators, detectors, etc.), and most significantly, the emergence of applications, such as neural networks, machine vision, and databases, that can make use of the capabilities of holographic 3-D memories. The theoretical upper limit on the storage density is $V / \lambda^{3}$, where $V$ is the volume of the hologram and $\lambda$ is the wavelength of the light. This limit is of the order of $10^{12} \mathrm{bits} / \mathrm{cm}^{3}$; however, in practical systems only $10^{9}-10^{10} \mathrm{bits} / \mathrm{cm}^{3}$ is achievable because of the finite numerical aperture of the optical system that transfers the data into the optical system and the dynamic range of the crystal. For example, $10^{3}$ can be superimposed at the same location, each hologram consisting of $10^{3} \times 10^{3}$ pixels, giving a total memory of $10^{9}$ bits per location. The practical usefulness of such a memory must be considered in light of the fact that electronic RAM chips

The authors are with the Department of Electrical Engineering, California Institute of Technology, Pasadena, California 91125.

Received 29 January 1993; revised manuscript received 21 October 1993.

0003-6935/94/173764-11\$06.00/0.

(1) 1994 Optical Society of America. currently under development have a storage density of $64 \times 10^{6}$ bits. $^{6} \quad$ Twenty such chips mounted on a single board could match the storage capability of the volume hologram. In order to build a mass storage medium that is not threatened by semiconductor memories, we must construct holographic memories that have a capacity much larger than $10^{9}$ bits.

In this paper we present a spatially multiplexed 3-D holographic storage scheme that we refer to as the 3-D holographic disk (HD). ${ }^{7}$ As in all spatial multiplexing schemes, the most crucial component of the system is the scanning mechanism that steers the readout mechanism to different locations on the disk. In our system, spatial multiplexing is done in a disk configuration with the rotation used to access different recording locations, as shown in Fig. 1. Two light beams (a signal beam and a reference beam) interfere inside the photorefractive crystal to create a phase grating by means of the photorefractive effect. We record multiple holograms at the same location by changing the reference-beam angle (angle multiplexing) or by changing the wavelengths of the reference and signal beams (wavelength multiplexing). Because of the Bragg-matching requirement of volume holograms, we can read out individual holograms by changing the direction of the reference beam (for angle multiplexing) or the wavelength of the reference beam (for wavelength multiplexing). We assume that the image beam is at normal incidence on the crystal.

The main result of this paper is the derivation of the storage capacity of 3-D HD's as limited by geometrical constraints. We show that a 3-D disk that is approximately $1.5 \mathrm{~cm}$ thick has a storage density approximately equal to $120 \mathrm{bits} / \mu \mathrm{m}^{2}$. Thus a $3-\mathrm{D}$ $\mathrm{HD}$ stores the equivalent of more than a 100 conventional two-dimensional disks of the same area. 


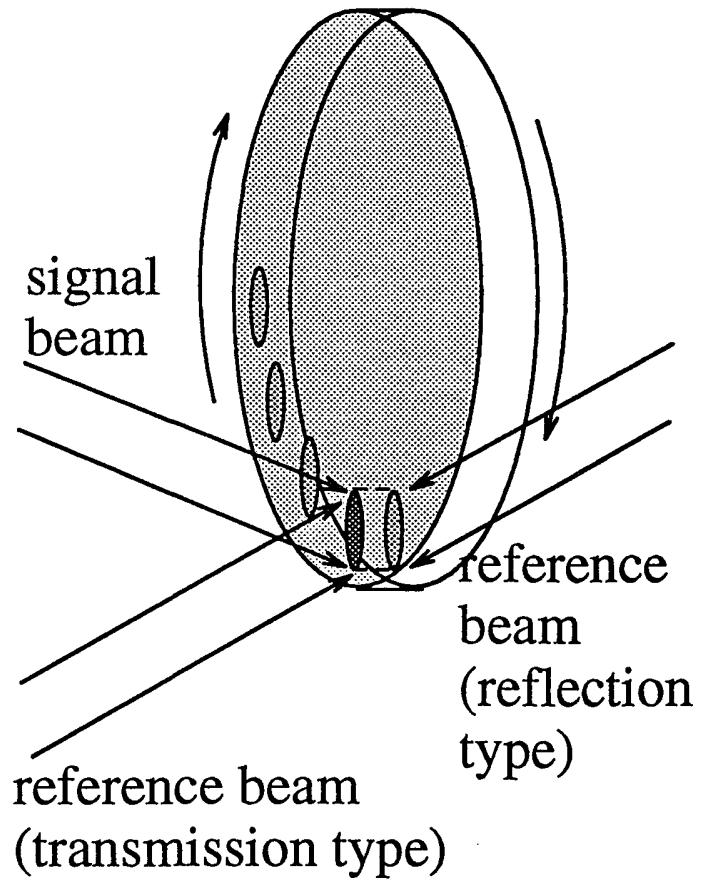

Fig. 1. Three-dimensional holographic disk.

\section{Angle-Multiplexed Holographic Disk}

Here we address the following question: What is the maximum number of bits $N$ that can be stored in a 3-D HD of area $A$ by using angle multiplexing? We show below that to maximize $N$ we must properly select the thickness of the $\mathrm{HD}(L)$, the magnification of the optical system that transfers the data to the disk, and the angles of incidence for the reference beam. In what follows we derive these optimum parameters. The limits to storage capacity in this paper are due to geometrical constraints. The dynamic range of the recording material imposes a limit on storage density independently. It turns out that the capacity caused by the geometric constraints is more restrictive than the material limitations in the 3-D HD system.

We can express $N$ as follows:

$$
N=N_{s} N_{\theta} N_{p}^{2} .
$$

In Eq. (1) $N_{s}$ is the number of separate locations on the disk where holograms are superimposed, $N_{\theta}$ is the number of holograms that are angularly multiplexed at the same location, and $N_{p}{ }^{2}$ is the number of pixels in each stored hologram. Below we derive an expression for each of the three quantities and then maximize their product with respect to the various parameters of the system.

\section{Maximum Number of Angularly Multiplexed Holograms}

We derive an expression for the maximum number of holograms $N_{\theta}$ that can be angularly multiplexed at a single location. In the following analysis we store data by recording either reflection or transmission holograms. The reference beam is a plane wave whose incident angle is $\theta_{R}$. The signal beam can be considered as a superposition of plane waves that spans a range of angles. To calculate $N_{\theta}$ we must first calculate $\Delta \theta_{R}$, the full width of the angular selectivity of each hologram, which we take to be the angular separation between adjacent holograms. An approximate expression for $\Delta \theta_{R}$ is ${ }^{8}$

$$
\Delta \theta_{R}=\frac{8 \lambda}{n \pi L} \frac{\cos \theta_{S}}{\left|\sin \left(\theta_{R}+\theta_{S}\right)\right|},
$$

where $\lambda$ is the wavelength, $L$ is the thickness of the hologram, $n$ is the index, and $\theta_{S}$ is the incident angle of the central plane-wave component of the signal beam (see Fig. 2). For transmission holograms $0<$ $\left|\theta_{R}\right|<\pi / 2$, and for reflection holograms $\pi / 2<$ $\left|\theta_{R}\right|<\pi$. The signal beam is assumed to be in the range $0<\left|\theta_{S}\right|<\pi / 2$.

Equation (2) is only an approximate estimate for the angular selectivity of the entire grating because different plane-wave components have different $\Delta \theta_{R}$. However, Eq. (2) is commonly used for setting the angular separation between reference-beam angles. The cross talk resulting when holograms are angularly multiplexed in this way has recently been calculated. ${ }^{9}$

To calculate the number of holograms that can fit into a range of reference-beam angles $\theta_{R}$ spanning from $\theta_{1}$ to $\theta_{2}$ (each hologram being separated from its adjacent holograms by a corresponding $\Delta \theta_{R}$ ), we observe that

$$
\left|\sin \left(\theta_{R}+\theta_{S}\right)\right| \Delta \theta_{R}=\frac{8 \lambda}{n \pi L} \cos \theta_{S}
$$

which is valid for all possible angles $\theta_{R}$. If we add together $N_{\theta}-1$ such equations, one for each value of $\theta_{R}$, and approximate the left-hand side of the summation by an integral, we obtain

$$
\int_{\theta_{1}}^{\theta_{2}}\left|\sin \left(\theta_{R}+\theta_{S}\right)\right| \mathrm{d} \theta_{R}=\frac{8 \lambda\left(N_{\theta}-1\right)}{n \pi L} \cos \theta_{S} .
$$

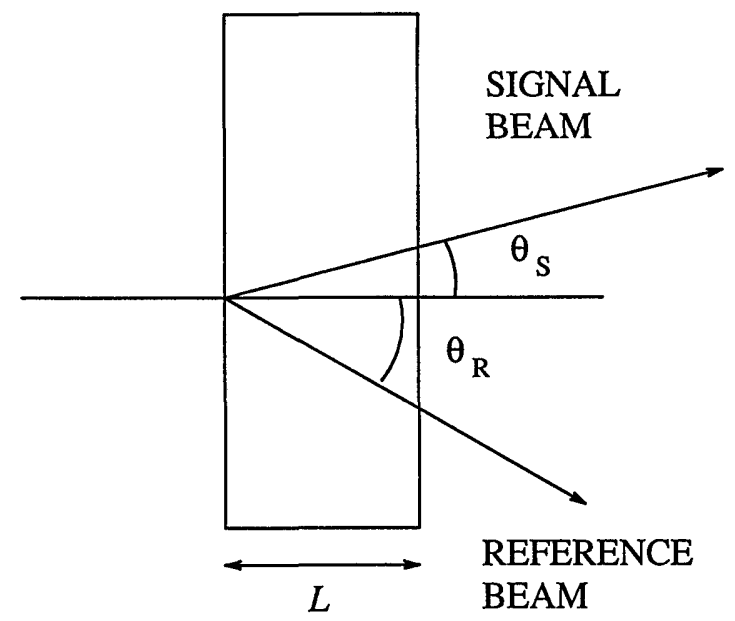

Fig. 2. Recording geometry. 
Solving for the number of reference angles, we get

$$
N_{0}=1+\left(\frac{n \pi L}{8 \lambda}\right) \frac{\left|\cos \left(\theta_{S}+\theta_{1}\right)-\cos \left(\theta_{S}+\theta_{2}\right)\right|}{\cos \theta_{S}}
$$

where it is assumed that either $0<\theta_{S}+\theta_{1}<\theta_{S}+$ $\theta_{2}<\pi$, or $-\pi / 2<\theta_{S}+\theta_{1}<\theta_{S}+\theta_{2}<0$. Physically this means that the reference beam is always to one side of the signal beam. The above calculations were carried out for angles inside the crystal. We can use Snell's law to convert to angles outside the crystal.

In what follows, we assume that the image beam has normal incidence $\left(\theta_{S}=0\right)$. In this case Eq. (5) becomes

$$
N_{\theta}=1+\left(\frac{n \pi L}{8 \lambda}\right)\left|\cos \theta_{1}-\cos \theta_{2}\right|,
$$

where we have $0<\theta_{1}<\theta_{2}<\pi / 2$ for transmission holograms. We can increase $N_{\theta}$ by a factor of 2 by recording a second set of angularly multiplexed holograms in the range $-\theta_{1}$ to $-\theta_{2}$, because the number of holograms that can be angularly multiplexed in the same range of angles is equal to the expression in Eq. (6). It is also possible to multiplex reflection and transmission holograms angularly at the same time, as shown in Fig. 3. Therefore, the geometric limit on the total number of holograms that can be superimposed in the same location is four times the expression in Eq. (6).

\section{Spatial Multiplexing}

The number of nonoverlapping spatial locations on a disk with $\operatorname{area} A$ is

$$
N_{s}=\frac{A}{a}=\frac{A}{w w^{\prime}},
$$

where $a=w \times w^{\prime}$ is the area of each location. To determine $w$ and $w^{\prime}$ we have to take into account the fact that the stored images can be in exact focus at only one plane in the volume of the crystal. As the thickness of the crystal increases, the area occupied

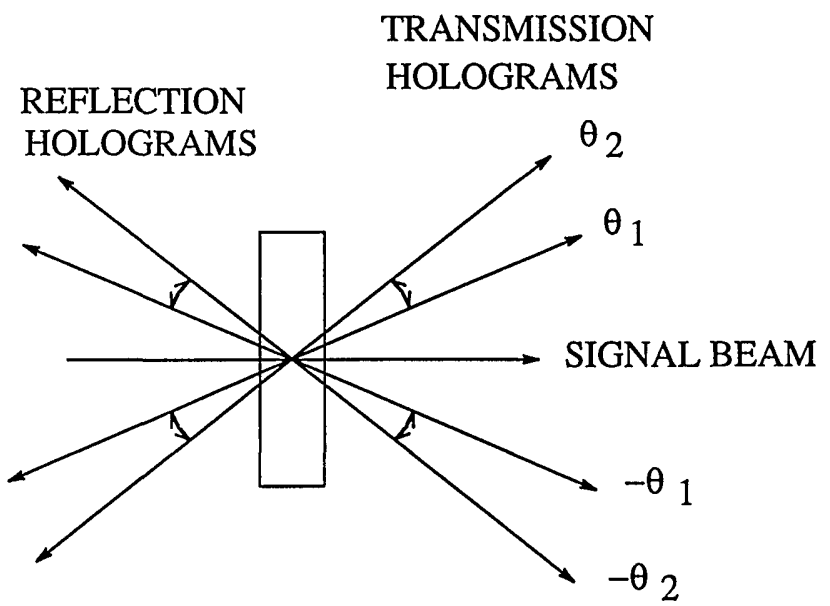

Fig. 3. Angular multiplexing by reflection and transmission holograms from both sides of the signal beam. by the defocused image at the surface of the hologram also increases. Moreover, the size of the area that is illuminated by the off-axis reference beam increases in one dimension as the crystal thickness and the angular sweep increase. We derive expressions for $w$ and $w^{\prime}$ with reference to the geometry of Fig. 4 . We assume that the images to be stored are at normal incidence and are focused at the middle of the crystal. We can calculate the extent of the defocused image on the surfaces by tracing the rays corresponding to the highest spatial frequency of the focused image. Let $\delta$ be the resolution or pixel spacing of the focused image. Then the maximum spatial frequency is approximately $1 / \delta$, corresponding to a diffracted plane wave traveling at an angle $\theta=\sin ^{-1}(\lambda / n \delta)$. We use the ray optics approximation to trace this maximum spatial frequency component and to obtain the size of the defocused image at the crystal faces:

$$
w=N_{p} \delta+L \tan \theta=N_{p} \delta+\frac{L}{\left[(n \delta / \lambda)^{2}-1\right]^{1 / 2}} .
$$

As we show in Fig. 4, in order for the reference beam to illuminate fully the volume of the crystal that the signal beam occupies, it must illuminate a width larger than $w$ in the direction of referencebeam sweep. From the geometry of Fig. 4 we see that this width is

$$
w^{\prime}=w+L \tan \theta_{2}
$$

The overall area that must be devoted to each record-

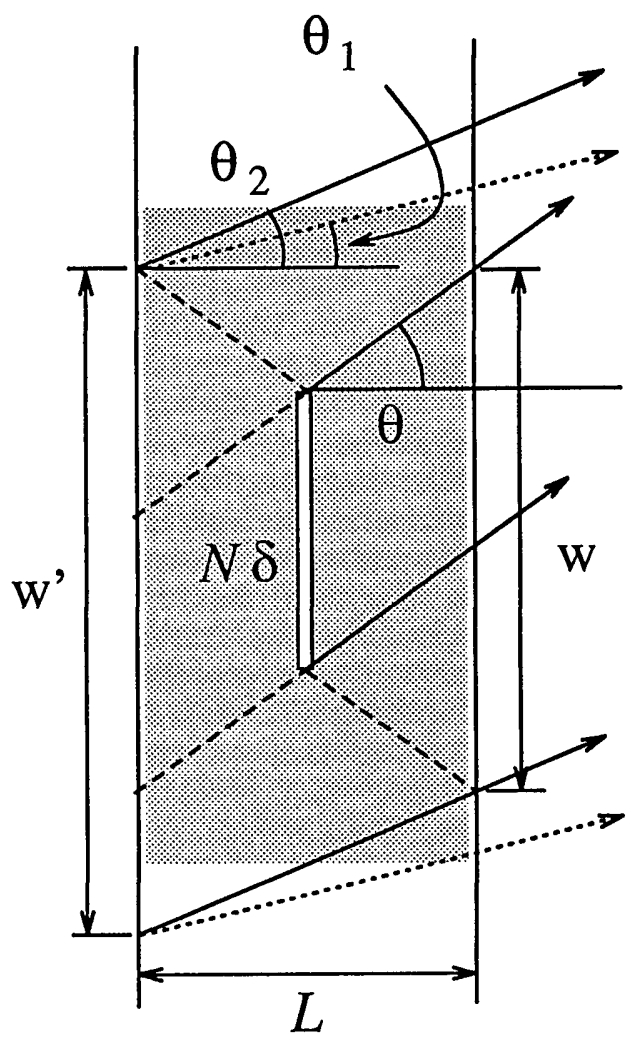

Fig. 4. Angle multiplexing: extra area taken up by defocusing and reference-beam angle change. 
ing location is therefore

$$
a=w w^{\prime}=w\left(w+L \tan \theta_{2}\right),
$$

where $w$ is given by Eq. (8).

Optimum $N_{p}, \theta_{1}$, and $\delta$

We can now write an expression for $N$, the total number of bits stored, by using Eqs. (7)-(10) and Eq. (1): with $\delta$ ) and set the derivative to zero. This yields the following equation for $y$ :

$$
y^{3}=c y+1 .
$$

The solution to this cubic equation is

$$
y=\sqrt[3]{\frac{1}{2}+\left(\frac{1}{4}-\frac{c^{3}}{27}\right)^{1 / 2}}+\sqrt[3]{\frac{1}{2}-\left(\frac{1}{4}-\frac{c^{3}}{27}\right)^{1 / 2}}
$$

$$
N=A N_{p}^{2} \frac{N_{\theta}}{w w^{\prime}}=A N_{p}^{2} \frac{1+\frac{n \pi L}{8 \lambda}\left(\cos \theta_{1}-\cos \theta_{2}\right)}{\left\{N_{p} \delta+\frac{L}{\left[(n \delta / \lambda)^{2}-1\right]^{1 / 2}}\right\}\left\{N_{p} \delta+\frac{L}{\left[(n \delta / \lambda)^{2}-1\right]^{1 / 2}}+L \tan \theta_{2}\right\}}
$$

We wish to maximize the above expression by optimally selecting $N_{p}, L, \theta_{1}, \theta_{2}$, and $\delta$, which are the parameters we can control.

First, we note that $N$ decreases monotonically as $\theta_{1}$ increases (in our analysis $0<\theta_{1}<\pi / 2$ ); therefore $\theta_{1}=0$ is the optimum value. However, because the angular selectivity is poor around $\theta_{1}=0$, in practice the minimum angle of the reference is set at $\theta_{1} \approx 10^{\circ}$ inside the crystal. Next we consider the optimum number of pixels $N_{p}$. Taking the derivative of $N$ with respect to $N_{p}$ shows that $N$ is a monotonically increasing function of $N_{p}$. This result confirms our theory because the increase in the disk area required to store the holograms because of defocusing and angular multiplexing can be thought of as an edge effect. The use of larger images implies fewer recording locations on the same disk area, and hence fewer edges. In practice $N_{p}$ is limited by the number of pixels of the spatial light modulator to approximately $N_{p}=1000$. For the rest of this section we consider $\theta_{1}$ and $N_{p}$ to be given and fixed.

The determination for the three remaining variables $\left(L, \theta_{2}\right.$, and $\left.\delta\right)$ is more difficult. We first consider the optimum pixel size $\delta$. For a given $L, N$ is maximized with respect to $\delta$ when $w$ is minimized with respect to $\delta$. To find the optimum $\delta$ we find it convenient to write $w$ as

$$
w=\frac{\lambda N_{p}}{n}\left[y^{3 / 2}+\frac{c^{3 / 2}}{\left(y^{3}-1\right)^{1 / 2}}\right],
$$

where

$$
\begin{aligned}
& y=\left(\frac{n \delta}{\lambda}\right)^{2 / 3}, \\
& c=\left(\frac{n L}{\lambda N_{p}}\right)^{2 / 3} .
\end{aligned}
$$

To minimize $w$ with respect to $\delta$, we differentiate $w$ with respect to $y$ (because $y$ increases monotonically which can be calculated for a given $L$ to yield the optimum $\delta$. Once $y$ is determined we can solve for the optimum pixel spacing $\delta_{o}$ :

$$
\delta_{o}=\frac{\lambda}{n} y^{3 / 2} .
$$

It can be shown that $\delta_{o}$ increases as $L$ increases.

The above solution, however, may not always be realizable. In any practical system, the minimum $\delta$ (denoted as $\delta_{\min }$ ) is limited by the imaging system to a value larger than the wavelength $\lambda$. If we use an imaging lens of $F$-number $z$, then the smallest resolvable spot is

$$
\delta_{\min }=\lambda \sqrt{\left(4 z^{2}+1\right)}
$$

which corresponds to the highest spatial frequency plane wave traveling at an angle

$$
\theta_{i}=\sin ^{-1}\left(\frac{\lambda}{\delta}\right)
$$

Inside the crystal, from Snell's law this becomes

$$
\begin{aligned}
\theta_{r} & =\sin ^{-1}\left(\frac{1}{n} \sin \theta_{i}\right), \\
& =\sin ^{-1}\left(\frac{\lambda}{n \delta_{\min }}\right) .
\end{aligned}
$$

Therefore the smallest resolvable spot size inside the crystal is also $\delta_{\min }$ as given by Eq. (17).

The lower bound for the size of $\delta$ is $\delta_{\min }$. If $L$ is too small, $\delta_{o}$ from Eq. (15) becomes less than $\delta_{\min }$. In that case we set $\delta=\delta_{\min }$. Because $\delta_{o}$ increases as $L$ increases, we can use Eqs. (13)-(16) to find the smallest $L$ for which $\delta_{o}$ is larger than $\delta_{\text {min }}$ :

$$
L_{\min }=\frac{\lambda N_{p}}{n}\left(\frac{y_{\min }{ }^{3}-1}{y_{\min }}\right)^{3 / 2}
$$


where

$$
y_{\min }=\left(\frac{n \delta_{\min }}{\lambda}\right)^{3 / 2}
$$

We will refer to the condition in which the optimum $\delta$ is less than $\delta_{\min }$ as the thin disk regime. If, in contrast, the optimum pixel spacing is larger than the resolution limit of the lens, this corresponds to the thick regime. For example, for $n=2.2, \lambda=500 \mathrm{~nm}$, $N_{p}=1000$, and using an $F / 3$ imaging lens (i.e., $z=3$ ), we get $\delta_{\min }=3.04 \mu \mathrm{m}$ and $L_{\min }=40.36 \mathrm{~mm}$. Note that $L_{\min }$ does not depend on $\theta_{2}$.

To summarize, if $L>L_{\min }$ (thick disk), we use $\delta=$ $\delta_{o}$ from Eq. (15), and if $L<L_{\min }$ (thin disk), we use $\delta=\delta_{\min }$ as given by Eq. (17).

\section{Optimum Thickness $L$}

Our problem is now reduced to maximizing $N$ with respect to the two remaining variables $L$ and $\theta_{2}$. We first treat $\theta_{2}$ as fixed and find the optimum $L$ that maximizes $N / A$. In the range $L<L_{\min }$ we use $\delta_{\min }$ as the optimum $\delta$, and we write $N / A$ from Eq. (11) as assuming of course that $L_{o}<L_{\min }$. If $L_{o}>L_{\min }$, this means that the optimum thickness is out of the thin regime where the analysis used to derive $L_{o}$ applies. Within the thin regime the maximum thickness $L$ occurs at the boundary because $N / A$ is monotonically increasing with $L$ for $L<L_{\text {min }}$. To obtain the overall optimum thickness $L$, we must compare the maximum obtained from this regime (i.e., $L \leq L_{\min }$ ) with the optimum thickness obtained from the thick regime $\left(L>L_{\min }\right)$ and finally select the thickness that yields the larger density $N / A$.

As an example, we continue with the previous example in which $\delta_{\min }=3.04 \mu \mathrm{m}$ (for a lens with an $F$-number of 3 ). If we take $\theta_{1}=10^{\circ}$ and $\theta_{2}=20^{\circ}$, we find $L_{o}$ to be $16.74 \mathrm{~mm}$, which is less than $L_{\min }=$ $40.36 \mathrm{~mm}$. Therefore, the solution obtained from the thin regime is the valid optimum thickness. Note that as $N_{p}$ increases so does $\alpha$, and therefore the expression in Eq. (27) increases. For large $N_{p}$, the maximum $N / A$ as given by Eq. (27) increases approximately linearly with $N_{p}$, or the square root of the total number of pixels $N_{p}^{2}$.

For $L>L_{\min }$, we can use Eq. (16) for $\delta$; using Eqs. (12)-(15) we can write Eq. (11) as

$$
N / A=\left(\frac{n}{\lambda}\right)^{2} \frac{1+\alpha c^{3 / 2}}{\left[y^{3 / 2}+\frac{c^{3 / 2}}{\left(y^{3}-1\right)^{1 / 2}}\right]\left[y^{3 / 2}+\frac{c^{3 / 2}}{\left(y^{3}-1\right)^{1 / 2}}+c^{3 / 2} \tan \theta_{2}\right]}
$$

$$
N / A=\frac{1}{\delta_{\min }^{2}} \frac{1+\alpha x}{(1+\beta x)(1+\gamma x)},
$$

where

$$
\begin{aligned}
& x=\frac{n L}{\lambda N_{p}}, \\
& \alpha=\frac{\pi N_{p}}{8}\left(\cos \theta_{1}-\cos \theta_{2}\right), \\
& \beta=\frac{1}{\left.y_{\min ^{3 / 2}}{ }^{3} y_{\min ^{3}}{ }^{3} 1\right)^{1 / 2}}, \\
& \gamma=\beta+\frac{\tan \theta_{2}}{y_{\min ^{3}}} .
\end{aligned}
$$

We can solve for the optimum $L$ by differentiating the expression in Eq. (22) with respect to $x$. The maximum $N / A$ turns out to be

$$
N / A=\frac{1}{\delta_{\min }^{2}} \frac{\alpha / \beta \gamma}{\left[\left(\frac{1}{\beta}-\frac{1}{\alpha}\right)^{1 / 2}+\left(\frac{1}{\gamma}-\frac{1}{\alpha}\right)^{1 / 2}\right]^{2}},
$$

which occurs at

$$
L=L_{0}=\frac{\lambda N_{p}}{n}\left\{-\frac{1}{\alpha}+\left[\left(\frac{1}{\beta}-\frac{1}{\alpha}\right)^{1 / 2}\left(\frac{1}{\gamma}-\frac{1}{\alpha}\right)\right]^{1 / 2}\right\},
$$

Equation (29) can be evaluated numerically to find the value of $L$ that maximizes $N / A$. We can also derive a relatively simple asymptotic expression (for large $L$ ) by observing that as $L \rightarrow \infty, y \rightarrow \sqrt{c}$. The asymptotic expression for $N / A$ is

$$
N / A \rightarrow\left(\frac{n}{\lambda}\right)^{2} \frac{\pi N_{p}\left(\cos \theta_{1}-\cos \theta_{2}\right)}{16 \tan \theta_{2}}\left(\frac{\lambda N_{p}}{n L}\right)^{1 / 2} .
$$

Expression (30) predicts that the density will decrease as the disk thickness becomes very large. This is confirmed by the numerical results we present in the following section.

\section{Optimum $\theta_{2}$ and Maximum Storage Density}

The final step in the optimization of the storage density $N / A$ consists of optimally selecting $\theta_{2}$. Because we cannot analytically derive the optimum angle, we resort to numerical methods. In Fig. 5 we plot Eq. (22) in the thin regime (solid curve) and Eq. (29) in the thick regime (dotted curve) as a function of $L$ for various values of $\theta_{2}$ by using the optimum value for $\delta$. The vertical dashed line indicates the transition from one regime to the other. The optimum values for $L$ and $\theta$ are those that yield the maximum density. The parameters used in plotting Fig. 5 are $\lambda=500 \mathrm{~nm}, N_{p}=1000, n=2.2$ (the index of refraction for $\mathrm{LiNbO}_{3}$ crystals), and $\theta_{1}=10^{\circ}$. Here $\theta_{2}=30^{\circ}$ is the maximum value for which $N / A$ is 
plotted because $27.04^{\circ}$ is the largest angle that can be supported inside the crystal (because of Snell's law) without resorting to the use of index-matching fluids.

From Fig. 5 we see that the maximum $N / A$ is obtained near $L=1.5 \mathrm{~cm}$ and increases monotonically with $\theta_{2}$ for the parameters we selected. In this case the optimum thickness is in the thin regime $\left(L_{o}<L_{\min }\right)$. Because it is not practical to use $\theta_{2}=$ $30^{\circ}$ inside the crystal (the critical angle is $27.04^{\circ}$ ), we get a realistic estimate for the achievable density by using $\theta_{2}=20^{\circ}$. The corresponding angle swing outside the crystal is then $22.5^{\circ}$ to $48.8^{\circ}$ (total angular swing of $26.3^{\circ}$ ), which is practically achievable. The maximum density $N / A$ is $29.3 \mathrm{bits} / \mu \mathrm{m}^{2}$, which is obtained for a crystal thickness of $L=16.74 \mathrm{~mm}$ by using $N_{\theta}=1306$ angularly multiplexed holograms. This density can be increased by a factor of 4 (giving us $N / A=117.2 \mathrm{bits} / \mu^{2}$ ) if we simultaneously record reflection and transmission holograms in the same reference-angle range from both sides of the signal beam, as shown in Fig. 3. The area for each recording location is $w \times w^{\prime}=4.3 \times 10.4 \mathrm{~mm}^{2}$. Figure 6 is a plot of the optimum density and also the number of angularly multiplexed holograms, $N_{\theta}$, as a function of $L$. For the thickness that yields maximum density, $N_{\theta}=1306$ holograms. Because more than 5000 holograms have been recorded and faithfully reproduced in lithium niobate, ${ }^{10}$ the geometric factors considered in this paper limit the recording more severely than the material dynamic range. As another example, if we record only 100 holograms at each location, then the optimum thickness is a little over $1 \mathrm{~mm}$ and the corresponding storage density is approximately $8.8 \mathrm{bits} / \mu \mathrm{m}^{2}$ (compared with approximately $30 \mathrm{bits} / \mu \mathrm{m}^{2}$ for the optimum design). This density can be increased by a factor of 4 , as we already described in Fig. 3.

\section{$N / A$ for various values of $\Theta_{2}$}

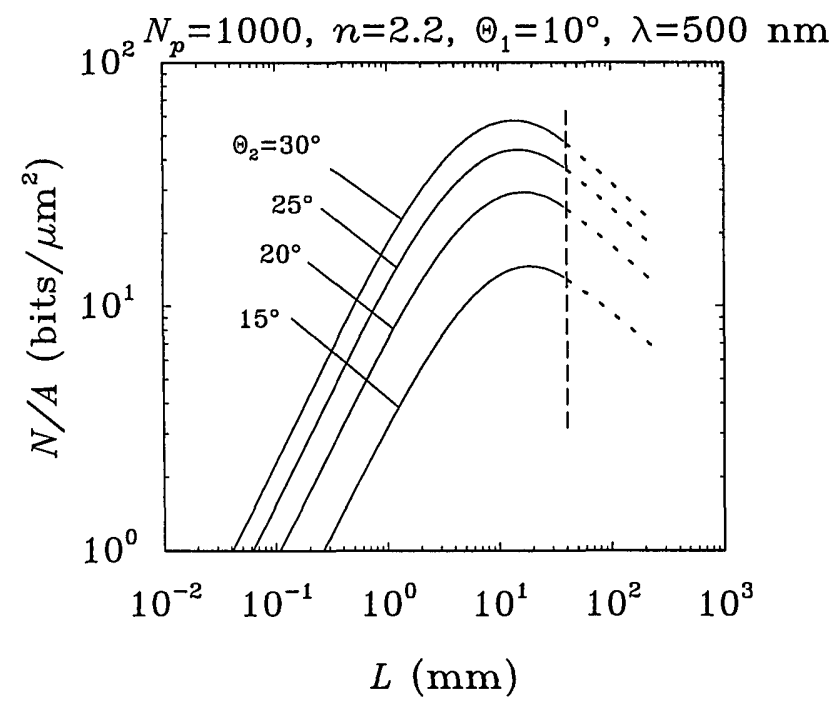

Fig. 5. Angle multiplexing: $N / A$ versus $L$ for various values of $\theta_{2}$.

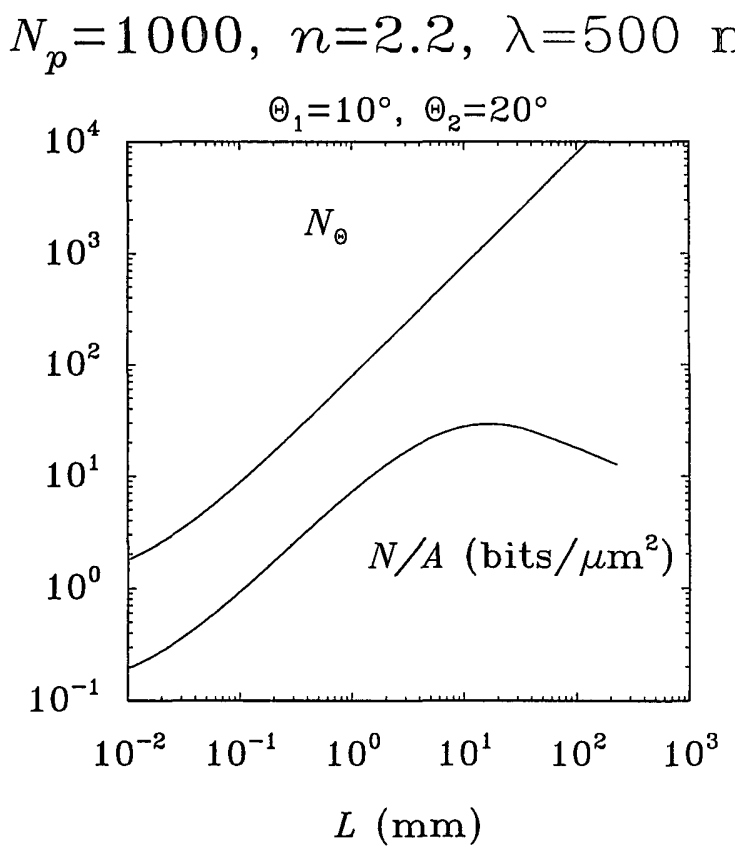

Fig. 6. Angle multiplexing: optimum $N / A$ (optimized with respect to $\delta$ ) and $N_{p}$ as functions of thickness $L$.

\section{Wavelength Multiplexing}

Wavelength multiplexing ${ }^{11,12}$ is an alternative method for multiplexing holograms in a single location on the $\mathrm{HD}$. In this section we calculate the capacity of a wavelength-multiplexed HD by using a similar derivation as for angular multiplexing. The number of bits that can be stored is expressed as

$$
N=N_{s} N_{\lambda} N_{p}^{2}
$$

where $N_{\lambda}$ is the number of wavelength-multiplexed holograms. We assume that the wavelength $\lambda$ sweeps from $\lambda_{1}$ to $\lambda_{2}$ with $\lambda_{1}<\lambda_{2}$.

For wavelength multiplexing, we again assume that the image is at normal incidence and focused at the middle of the crystal (Fig. 7), and that the reference beam is counterpropagating with the image beam also at normal incidence. In this case the problem of imaging defocusing at the crystal surface is the same, and we get Eq. (8) for the width $w$ as before. However, because the reference beam is colinear with the signal beam for all wavelengths, there is no extra width taken up by the $L \tan \theta_{2}$ term in the expression for $w^{\prime}$ in Eq. (9). In contrast, as $\lambda$ sweeps through $\lambda_{1}$ to $\lambda_{2}, w$ changes. For any choice of $\delta$, the largest $w$ is for $\lambda=\lambda_{2}$. Therefore we have

$$
N_{s}=\frac{A}{w^{2}}
$$

where

$$
w=N_{p} \delta+\frac{L}{\left[\left(n \delta / \lambda_{2}\right)^{2}-1\right]^{1 / 2}}
$$

is a function of $\lambda_{2}$. 


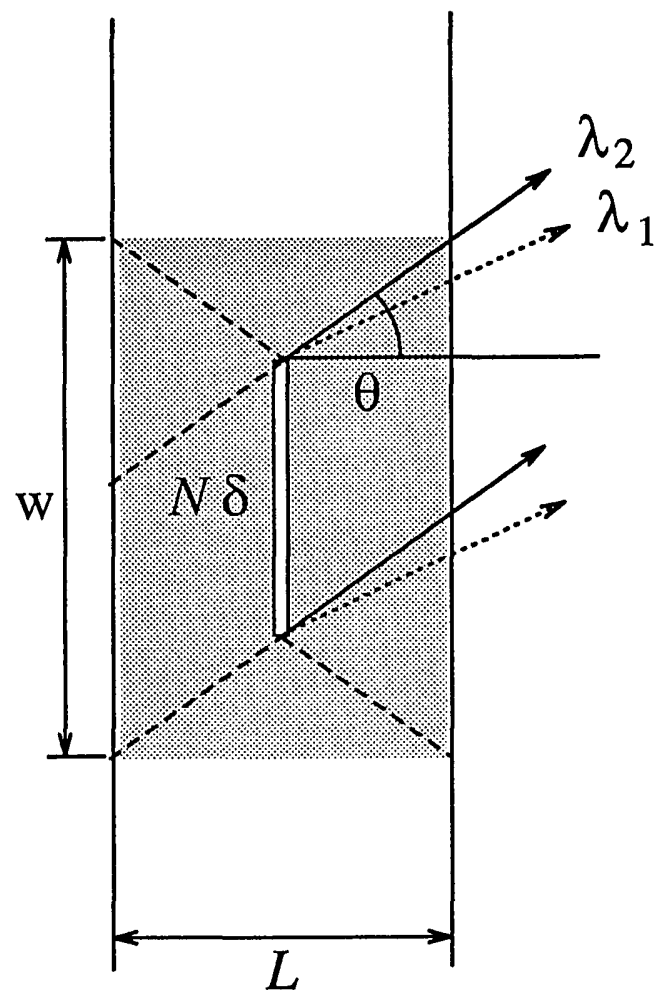

Fig. 7. Wavelength multiplexing: extra area taken up because of defocusing and wavelength change.

To find $N_{\lambda}$, we note that the half-width of the (frequency) selectivity $\Delta v$ is $^{8}$

$$
\Delta \nu=\frac{v_{c}}{n L},
$$

where $v_{c}$ is the speed of light in vacuum. As $\lambda$ sweeps over $\lambda_{1}$ to $\lambda_{2}$, the number of wavelength-multiplexed holograms that can be stored is therefore

$$
N_{\lambda}=1+\frac{\nu_{1}-\nu_{2}}{2 \Delta \nu}=1+\frac{n L}{2}\left(\frac{1}{\lambda_{1}}-\frac{1}{\lambda_{2}}\right),
$$

where we take the separation between adjacent holograms to be full-width $2 \Delta \nu$. Using Eqs. (32), (33), and (35), we then have

$$
N=A N_{p}^{2} \frac{1+\frac{n L}{2}\left(\frac{1}{\lambda_{1}}-\frac{1}{\lambda_{2}}\right)}{\left\{N_{p} \delta+\frac{L}{\left[\left(n \delta / \lambda_{2}\right)^{2}-1\right]^{1 / 2}}\right\}^{2}} .
$$

Optimum $N_{p}, \lambda_{1}$, and $\delta$

We now want to maximize $N$ with respect to $N_{p}, L, \lambda_{1}$, $\lambda_{2}$, and $\delta$. As before, $N$ increases monotonically with $N_{p}$, which is limited by the spatial light-modulator to approximately 1000 . $N$ also increases as the minimum wavelength $\lambda_{1}$ decreases. This will be limited by the shortest usable wavelength we can get out of a tunable laser, the spectral sensitivity of the material, or both. For the remainder of this section, we will assume that $N_{p}$ and $\lambda_{1}$ are given and fixed.

The three remaining parameters $\lambda_{2}, L$, and $\delta$ are more complicated. We first take $L$ and $\lambda_{2}$ as fixed, and we find the optimum $\delta$. Considering $N / A$ as a function $\delta$, we find as before that the maximum $N / A$ is obtained when $w$ is minimized with respect to $\delta$. We then get the same set of equations as Eqs. (13)-(16), except with $\lambda$ replaced by $\lambda_{2}$. We also have the same $\delta_{\min }$ and $L_{\min }$ (with $\lambda$ replaced by $\lambda_{2}$ ) conditions as given by Eqs. (17) and (20), respectively. Note that both $\delta_{\min }$ and $L_{\min }$ scale linearly with wavelength (because $y_{\min }$ depends only on $n$ and $z$, the $F$-number of the imaging lens). It should be emphasized that $\delta_{\min }$ is the resolution of the imaging system that uses wavelength $\lambda_{2}$. The resolution of the system that uses $\lambda_{1}$ (which is less than $\lambda_{2}$ ) is of course better.

In summary, if $L>L_{\min }$, we use $\delta=\delta_{o}$ from Eq. (15); otherwise we use $\delta=\delta_{\min }$ as defined in Eq. (17). In these equations $\lambda$ is replaced by $\lambda_{2}$. As an example, for $\lambda_{2}=540 \mathrm{~nm}$ and an imaging lens with an $F$-number of 3 , we have $\delta_{\min }=3.28 \mu \mathrm{m}$ and $L_{\min }=$ $43.59 \mathrm{~mm}$. For $\lambda_{2}=750 \mathrm{~nm}$, these become $\delta_{\min }=$ $4.56 \mu \mathrm{m}$ and $L_{\min }=60.54 \mathrm{~mm}$.

\section{Optimum $L$ and $\lambda_{2}$}

We now find the optimum thickness $L$ that maximizes $N / A$. In the thin regime $\left(L<L_{\min }\right)$ we take $\delta=\delta_{\min }$, and we write $N / A$ as

$$
N / A=1 \frac{1}{\delta_{\min }^{2}} \frac{1+\alpha x}{(1+\beta x)^{2}},
$$

where

$$
\begin{aligned}
& x=\frac{n L}{\lambda_{2} N_{p}}, \\
& \alpha=\frac{N_{p}}{2}\left(\frac{\lambda_{2}}{\lambda_{1}}-1\right), \\
& \beta=\frac{1}{y_{\min ^{3 / 2}}\left(y_{\left.\min ^{3}-1\right)^{1 / 2}}\right.} .
\end{aligned}
$$

By differentiating the expression in Eq. (37) with respect to $x$, we find the maximum $N / A$ to be

$$
N / A=\frac{1}{\delta_{\min }^{2}} \frac{\alpha^{2}}{4 \beta(\alpha-\beta)},
$$

which occurs at

$$
L=L_{o}=\frac{\lambda_{2} N_{p}}{n}\left(-\frac{2}{\alpha}+\frac{1}{\beta}\right) .
$$

For example, for $\lambda_{1}=500 \mathrm{~nm}, \lambda_{2}=540 \mathrm{~nm}$ $\left(\lambda_{2} / \lambda_{1}=1.08\right), n=2.2$, and $N_{p}=1000$, we get $L_{o}=$ $43.82 \mathrm{~mm}$. If $\lambda_{2}$ increases to $750 \mathrm{~nm}, L_{o}$ increases to $60.88 \mathrm{~mm}$. In both cases $L_{o}$ is larger than $L_{\min }(43.59$ $\mathrm{mm}$ and $60.54 \mathrm{~mm}$, respectively). This means that 
there is no maximum in the thin regime, and that therefore in the range $L<L_{\min }, N / A$ is monotonically increasing with $L$. In this case we would select the boundary value $\left(L_{\min }\right)$ for the best thickness obtainable from the thin regime. Notice that for wavelength-multiplexed storage, the optimum thickness of the disk can become large. Even though we are not considering materials issues in this paper, we should point out that the useful thickness of the material in practice can be limited by absorption. In some materials (e.g., lithium niobate) it is possible to reduce the absorption by the proper preparation of the material (e.g., by the adjustment of the dopant and reduction-oxidation level). The reduced absorption will typically reduce the recording speed of the material for a given light intensity. Therefore, when materials considerations are included in the design process, this trade-off between speed and density will emerge.

In the thick regime, $L>L_{\min }$, we use Eqs. (15) and (16) with $\lambda$ replaced by $\lambda_{2}$ to obtain $\delta$, and we write $N / A$ as

$$
N / A=\left(\frac{n}{\lambda_{2}}\right)^{2} \frac{1+\alpha c^{3 / 2}}{\left[y^{3 / 2}+\frac{c^{3 / 2}}{\left(y^{3}-1\right)^{1 / 2}}\right]^{2}},
$$

where $\alpha$ is given by Eq. (39). As before, as $L \rightarrow \infty$, $y \rightarrow \sqrt{c}$. For wavelength multiplexing, however, the asymptotic behavior of $N / A$ is different. As $L \rightarrow \infty$, $N / A$ saturates and approaches

$$
N / A \rightarrow \frac{n^{2} N_{p}}{8} \frac{1}{\lambda_{2}}\left(\frac{1}{\lambda_{1}}-\frac{1}{\lambda_{2}}\right) .
$$

Thus for the range $L>L_{\min }, N / A$ also increases monotonically with $L$.

Note that the saturation value of $N / A$ increases as $N_{p}$ increases and $\lambda_{1}$ decreases. In addition, for any choice of $\lambda_{2} / \lambda_{1}$, we have

$$
\frac{1}{\lambda_{2}}\left(\frac{1}{\lambda_{1}}-\frac{1}{\lambda_{2}}\right) \leq \frac{1}{4 \lambda_{1}^{2}},
$$

with equality at

$$
\frac{\lambda_{2}}{\lambda_{1}}=2
$$

Thus, even if it is practical to have a light source with such a large range of wavelength tunability, the optimum setting for $\lambda_{2} / \lambda_{1}$ for us to obtain maximum saturation density is 2 (provided we use the same $\lambda_{1}$ ). For practical systems $\lambda_{2} / \lambda_{1}$ is smaller than 2 , and in this range the saturation value of $N / A$ increases as $\lambda_{2} / \lambda_{1}$ increases. In the case in which $\Delta \lambda=\lambda_{2}-$ $\lambda_{1} \ll \lambda_{1}\left(\lambda_{2} \approx \lambda_{1}\right)$, the saturation value given in relation (44) is approximately

$$
N / A \approx \frac{n^{2} N_{p}}{8} \frac{\Delta \lambda}{\lambda_{1}^{3}},
$$

which is proportional to $\Delta \lambda$.

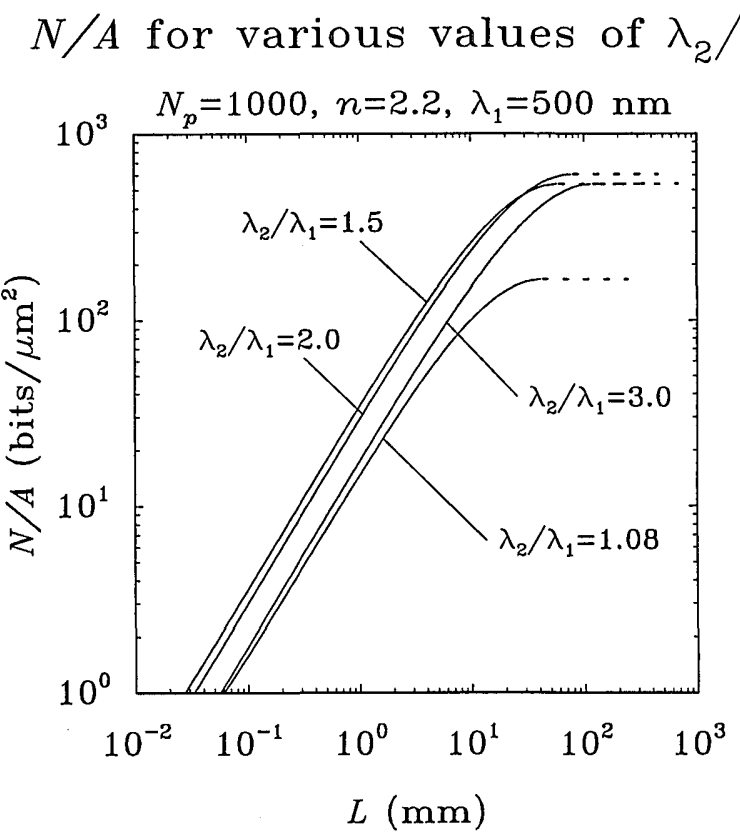

Fig. 8. Wavelength multiplexing: optimum $N / A$ (optimized with respect to $\delta$ ) as a function of $L$ for various values of $\lambda_{2} / \lambda_{1}$.

In practice, the range of usable wavelengths is determined by the laser system. For instance, dye lasers can be tuned in the range from 370 to $890 \mathrm{~nm}$, which gives us a $\lambda_{2} / \lambda_{1}$ of 2.40 , in excess of the optimum $\lambda_{2} / \lambda_{1}=2$ requirement. It should be noted, however, that it is necessary for us to use several different dyes to achieve this range of wavelengths. For a typical broadband laser dye such as Coumarin 6 , the range is from 510 to $550 \mathrm{~nm}$, which only gives us a $\lambda_{2} / \lambda_{1}$ of 1.08. For Ti:sapphire lasers the range is 600 to $1025 \mathrm{~nm}$, which gives us a $\lambda_{2} / \lambda_{1}$ of 1.48 .

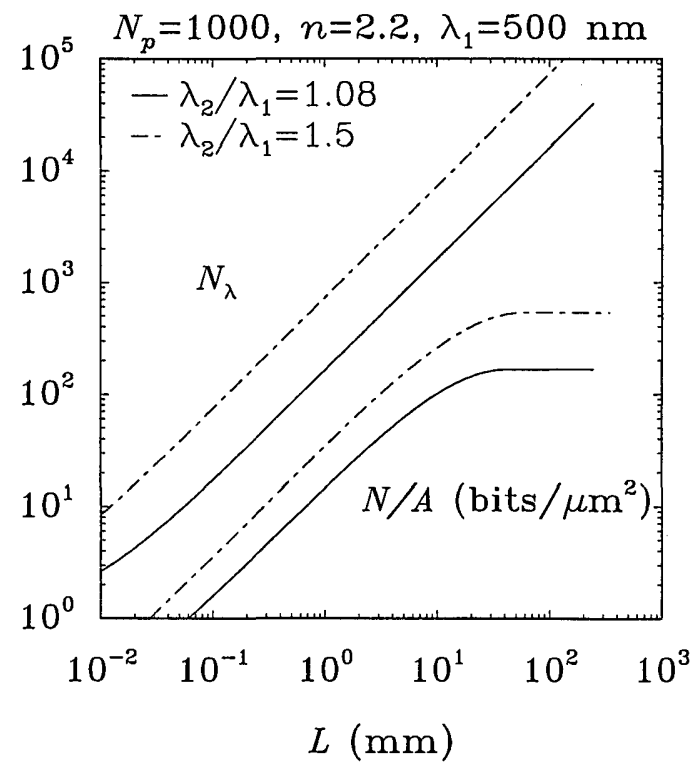

Fig. 9. Wavelength multiplexing: optimum $N / A$ and $N_{\lambda}$ as functions of thickness $L$ for $\lambda_{2} / \lambda_{1}=1.08$ and $\lambda_{2} / \lambda_{1}=1.5$. 


\begin{tabular}{lrr}
\hline & & Multiplexing \\
\cline { 2 - 3 } \multicolumn{1}{c}{ Parameters } & \multicolumn{1}{c}{ Angle } & Wavelength \\
\hline Index of refraction & $n=2.2$ & $n=2.2$ \\
Number of pixels & $N_{p}^{2}=10^{6}$ & $N_{p}^{2}=10^{6}$ \\
Wavelength & $\lambda=500 \mathrm{~nm}$ & $\lambda_{1}=500 \mathrm{~nm}, \lambda_{2}=540 \mathrm{~nm}$ \\
Angles & $\theta_{1}=10^{\circ}, \theta_{1}=20^{\circ}$ & - \\
Pixel size & $\delta_{\min }=3.04 \mu \mathrm{m}$ & $\delta_{\min }=3.28 \mu \mathrm{m}$ \\
Critical thickness & $L_{\min }=40.36 \mathrm{mn}$ & $L_{\min }=43.59 \mathrm{~mm}$ \\
Optimum thickness & $L_{o}=16.74 \mathrm{~mm}$ & $L_{o} \approx 30 \mathrm{~mm}$ \\
Maximum density & $N / A=4 \times 29.3 \mathrm{bits} / \mu \mathrm{m}^{2}$ & $N / A=166.0 \mathrm{bits} / \mu \mathrm{m}^{2}$ \\
Number of holograms & $N_{\theta}=1306$ & $N_{\lambda} \approx 5000$ \\
\hline
\end{tabular}

aThe $f$-number of the imaging lens is 3.

As a specific example, consider the case in which $N_{p}=1000, n=2.2$, and $\lambda_{1}=500 \mathrm{~nm}$. We plot $N / A$ as a function of $L$ (where $N / A$ has been optimized with respect to $\delta$ ) for various values of $\lambda_{2} / \lambda_{1}$. The result is shown in Fig. 8 . We see that $N / A$ saturates for large $L$ (near $5 \mathrm{~cm}$ ) as we expected, and the saturation value is largest for $\lambda_{2} / \lambda_{1}=2$. In Fig. 9, we plot $N / A$ and $N_{\lambda}$ as functions of $L$ for $\lambda_{2} / \lambda_{1}=1.08$ and $\lambda_{2} / \lambda_{1}=1.5$, and we use the same $N_{p}, n$, and $\lambda_{1}$.
For $\lambda_{2} / \lambda_{1}=1.08 N / A$ approaches $166.0 \mathrm{bits} / \mu \mathrm{m}^{2}$, whereas for $\lambda_{2} / \lambda_{1}=1.5 N / A$ approaches 537.8 bits $/ \mu \mathrm{m}^{2}$.

\section{Storage Density and Optimum $\lambda_{2}$ for Thin Disks}

The point where $L$ causes $N / A$ to reach saturation is of the order of $5 \mathrm{~cm}$. At this thickness, it becomes questionable about what we mean by a disk. In practice it may be desirable or necessary (e.g., because

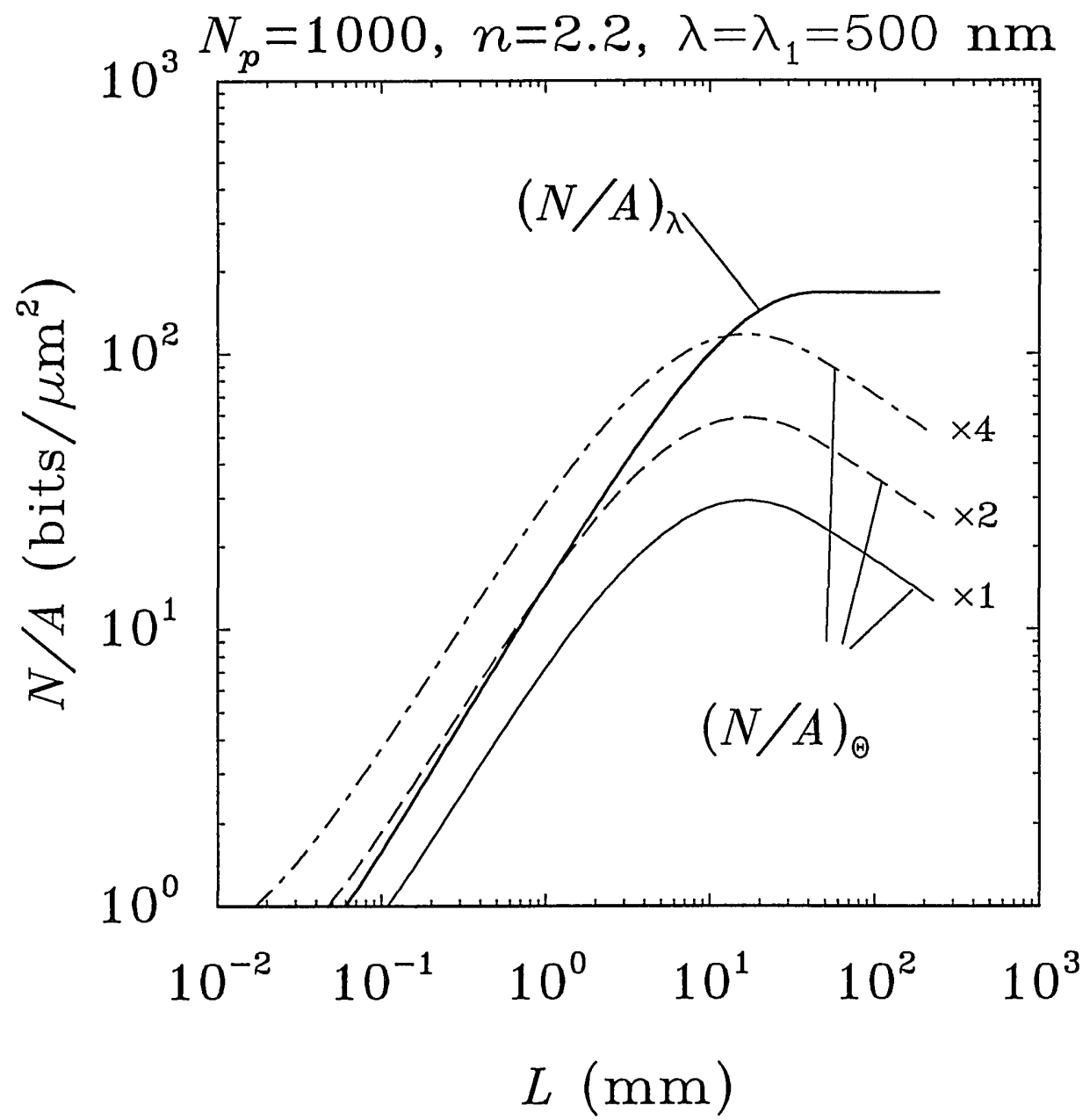

Fig. 10. Comparison of angle multiplexing and wavelength multiplexing. Here $\lambda_{2} / \lambda_{1}=1.08, \theta_{1}=10^{\circ}$, and $\theta_{2}=20^{\circ}$. The density when angle multiplexing is used is denoted by $(N / A)_{\theta}$, and the density when wavelength multiplexing is used is denoted by $(N / A)_{\lambda}$. 
of absorption) to keep the thickness small. In this case we are in the $L<L_{\min }$ range (even though $L_{o}$ may be larger than $L_{\min }$ ), and $N / A$ is given by Eq. (37). We can approximate Eq. (37) by

$$
N / A \approx \frac{n}{2 \delta_{\min }^{2}}\left(\frac{1}{\lambda_{1}}-\frac{1}{\lambda_{2}}\right) L \propto \frac{1}{\lambda_{2}^{2}}\left(\frac{1}{\lambda_{1}}-\frac{1}{\lambda_{2}}\right),
$$

if we assume that

$$
\alpha x \gg 1 \gg \beta x .
$$

In relation (49) $\alpha=40$ for $\lambda_{2} / \lambda_{1}=1.08$ and $\alpha=250$ for $\lambda_{2} / \lambda_{1}=1.5$, while $\beta=5.60 \times 10^{-3}$ in both cases, so the condition is satisfied.

If we limit $x$ and hence the disk thickness $L$ to the range required by relation (49), the optimum $\lambda_{2}$ can be found by taking the derivative of the expression in relation (48) with respect to $\lambda_{2}$ and setting it to zero. In this case it is easy to show that the maximum $N / A$ occurs for $\lambda_{2} / \lambda_{1}=1.5$ (again assuming that we are using the same $\lambda_{1}$ ), which is close to the value provided by Ti:sapphire lasers. Therefore, in this case the density does not increase indefinitely with $\Delta \lambda$.

Finally, we can also calculate the knee of the $N / A$ curve, which we define as the point where the expression given by Eq. (47) reaches the saturation value. This is given by

$$
L=L_{K}=\frac{n N_{p} \delta_{\min }^{2}}{4 \lambda_{2}}=\frac{4 z^{2}+1}{4} n \lambda_{2} N_{p},
$$

which is proportional to $\lambda_{2}$. For $\lambda_{2} / \lambda_{1}=1.08, L_{K}=$ $11.0 \mathrm{~mm}$, which gives us $N / A=106.5 \mathrm{bits} / \mu \mathrm{m}^{2}$ and $N_{\lambda}=1794$. For $\lambda_{2} / \lambda_{1}=1.5, L_{K}=15.3 \mathrm{~mm}$, which gives us $N / A=34.46 \mathrm{bits} / \mu \mathrm{m}^{2}$ and $N_{\lambda}=11,221$. In both cases $L_{K}$ is less than $L_{\min }$, and the corresponding values of $N / A$ are slightly over half of the saturation values for $N / A$ (i.e., approximately a $3-\mathrm{dB}$ drop).

\section{Discussion and Conclusions}

The values for the various parameters discussed herein are summarized in Table 1 , and the storage densities $N / A$ are plotted in Fig. 10, where we denote the densities of angle multiplexing and wavelength multiplexing by $(N / A)_{\theta}$ and $(N / A)_{\lambda}$, respectively.

In Fig. 10 the curve for $(N / A)_{\theta}$ that uses just the angle range from $\theta_{1}$ to $\theta_{2}$ (either as transmission or reflection holograms) is marked as $\times 1$. We see that it is approximately a factor of 2 smaller than $(N / A)_{\lambda}$. However, if we angle multiplex from both sides of the signal beam, $(N / A)_{\theta}$ increases by a factor of 2 (denoted by the $\times 2$ curve in Fig. 10). If we further record both reflection and transmission holograms (as in Fig. 3), this increases by a factor of 4 (denoted by the $\times 4$ curve in Fig. 10). In this case $(N / A)_{\theta}$ becomes larger than $(N / A)_{\lambda}$ until $L$ reaches approximately $12.5 \mathrm{~mm}$, where both $(N / A)_{\lambda}$ and $(N / A)_{\theta}$ are approximately $115 \mathrm{bits} / \mu \mathrm{m}^{2}$.

One might ask whether it is possible to achieve higher density by recording in the Fourier plane instead of the image plane. It turns out that the storage density is the same. This is because the space-bandwidth product is a constant. Specifically, consider an image of extent $a=N_{p} \delta$, where $\delta$ is the pixel spacing and $N_{p}$ is the number of pixels along one dimension. Let $b$ be the extent of the Fourier transform of this image (by a lens of focal length $F$ ), and let $1 / \delta^{\prime}$ be the highest spatial frequency of the Fourier transform. Then within the paraxial approximations,

$$
N_{p}=\frac{a}{\delta}=\frac{b}{\delta^{\prime}}
$$

This shows that recording in either the image plane or the Fourier plane will give the same minimum width.

If we record holograms at off-image or off-Fourier planes, the required width $w$ increases. However, it is sometimes desirable to do this for the purpose of noise, image quality, and alignment sensitivity. ${ }^{13}$ The trade-off between these requirements and storage density will have to be considered in the design of a practical system.

We have derived the optimum conditions for achieving the maximum storage density of a 3-D HD by using either angle multiplexing or wavelength multiplexing. Such optimally designed disks can store information with area densities of more than 100 $\mathrm{bits} / \mu \mathrm{m}^{2}$ and with disk thicknesses of approximately $1 \mathrm{~mm}$. However, the limits to storage density derived in this paper are only due to the geometry of the system. The storage density can also be limited by noise (cross talk, detector noise, media defects, etc.) and by the limited dynamic range of the recording medium. These limits to $N / A$ prove less restrictive than the geometric limits derived here. ${ }^{14}$ This is supported by recent experiments ${ }^{15}$ in which 1000 holograms were superimposed and reconstructed with an extremely low probability of error in a lithium niobate crystal that was $1 \mathrm{~cm}$ thick. Of course, given a minimum usable diffraction efficiency (from noise considerations), we find that the maximum number of holograms decreases as the number of pixels per hologram increases. This is because the amount of light diffracted for each pixel is less when there are more pixels per hologram. However, the parameters of this experiment $(320 \times 220$ instead of $1000 \times 1000$ pixels per hologram) were not too far from the parameters we used in deriving our results, and the dynamic range of the crystal was nowhere close to being exhausted.

The support of the U.S. Air Force Office of Scientific Research for this work is gratefully acknowledged. We also thank K. Curtis and D. Marx for many helpful discussions.

\section{References}

1. P. J. Van Heerden, "Theory of optical information storage in solids," Appl. Opt. 2, 393-400 (1963).

2. K. Bløtekjaer, "Limitations on holographic storage capacity of 
photochromic and photorefractive media," Appl. Opt. 18, 57-67 (1979).

3. D. von der Linde and A. M. Glass, "Photorefractive effects for reversible holographic storage of information," Appl. Phys. 8, 85-100 (1975).

4. J.-P. Huignard, J.-P. Herriau, and F. Micheron, "Coherent selective erasure of superimposed volume holograms in $\mathrm{LiNbO}_{3}$," Appl. Phys. Lett. 26, 256-258 (1975).

5. L. d'Auria, J.-P. Huignard, C. Slezak, and E. Spitz, "Experimental holographic read-write memory using 3-D storage," Appl. Opt. 13, 808-818 (1974).

6. Y. Nakagome, H. Tanaka, K. Takeuchi, E. Kume, Y. Watanabe, T. Kaga, Y. Kawamoto, F. Murai, R. Izawa, D. Hisamoto, T. Kisu, T. Nishida, E. Takeda, and K. Itoh, "An experimental 1.5-V 64-Mb DRAM," IEEE J. Solid-State Circuits 26, 465472 (1991).

7. D. Psaltis, "Parallel optical memories," Byte 17, 179-182 (1992).

8. H. Kogelnik, "Coupled wave theory for thick hologram gratings," Bell Syst. Tech. J. 48, 2909-2947 (1969).

9. C. Gu, J. Hong, I. McMichael, R. Saxena, and F. H. Mok, "Cross talk limited storage capacity of volume holographic memory," J. Opt. Soc. Am. A 9, 1-6 (1992).
10. F. H. Mok, "Applications of holographic storage in lithium niobate," in Annual Meeting, Vol. 23 of 1992 OSA Technical Digest Series (Optical Society of America, Washington, D.C., 1992), paper WE1.

11. F. T. S. Yu, S. D. Wu, A. W. Mayers, and S. M. Rajan, "Wavelength multiplexed reflection matched spatial filters using $\mathrm{LiNbO}_{3}$," Opt. Commun. 81, 343-347 (1991).

12. G. A. Rakuljic, V. Leyva, and A. Yariv, "Optical-data storage by using orthogonal wavelength-multiplexed volume holograms," Opt. Lett. 17, 1471-1473 (1992).

13. H.-Y. Li, "Photorefractive 3-D disks for optical data storage and artificial neural networks," Ph.D. dissertation (California Institute of Technology, Pasadena, Calif., 1994).

14. K. Curtis, G. W. Burr, and D. Psaltis, "Comparison of anglemultiplexed, wavelength-multiplexed, and phase-coded holographic memories," in Annual Meeting, Vol. 16 of 1993 Technical Digest Series (Optical Society of America, Washington, D.C., 1993), paper TuH3.

15. F. Mok, D. Psaltis, and G. Burr, "Spatial and angle multiplexed holographic random access memory," in Photonics for Computers, Neural Networks, and Memories, S. T. Kowel, W. J. Miceli, and J. A. Neff, eds., Proc. Soc. Photo-Opt. Instrum. Eng. 1773, 334-345 (1992). 